\title{
Efecto protector del aceite de plukenetia volubilis (sacha inchi) en la depresión inducida de ratones albinos
}

\author{
Protective effect of plukenetia volubilis (sacha inchi) oil \\ on induced depression of albino mice
}

Herencia-Anaya Melissa ${ }^{1, a}$, Mendoza-Yaranga Elizabeth ${ }^{1, a}$, Cáceres-Bellido Fermín ${ }^{1, a, b}$.

1. Universidad Nacional San Luis Gonzaga de Ica, Perú.

a. Médico Cirujano.

b. Cirujano General.

\section{Correspondencia:}

Herencia Anaya, Melissa Lorena. Número de celular: 985566837 Correo Electrónico:

melo.27.92@gmail.com

\section{Contribuciones De Autoría:}

HAM, MYE, CBF participaron en el diseño del estudio, el análisis de los datos, revisaron críticamente el artículo y aprobaron la versión final.

Conflicto De Intereses: No declarados.

Financiamiento: Autofinanciado.

\section{Como Citar}

Herencia-Anaya Melissa, Mendoza-Yaranga Elizabeth, Cáceres-BeIlido Fermín. Efecto protector del aceite de plukenetia volubilis (sacha inchi) en la depresión inducida de ratones albinos. Rev méd panacea 2018;7(1): 4-8

Recibido: 03 - 03 - 2018

Aceptado: 20 - 03 - 2018

Publicado: 23 - 04 - 2018

\section{RESUMEN}

Objetivo: Evaluar el efecto protector del aceite de Plukenetia volubilis (Sacha Inchi) en la depresión inducida a ratones albinos. Materiales y métodos: Los ratones fueron divididos en 4 grupos y recibieron durante 10 días las siguientes sustancias: Grupo $N^{\circ} 01(n=6)$ Vehículo $5 \mathrm{ml} / \mathrm{Kg} / 12 \mathrm{~h}$, Grupo $N^{\circ} 02(n=6)$ : Fluoxetina $10 \mathrm{mg} / \mathrm{Kg} / 24$ horas, Grupo $N^{\circ} 03(\mathrm{n}=6)$ : Aceite de sacha inchi $1 \mathrm{~g} / \mathrm{kg} / 12$ horas, Grupo No $04(\mathrm{n}=6)$ : Aceite de sacha inchi $3 \mathrm{~g} / \mathrm{kg} / 12$ horas. Luego, fueron sometidos a la prueba de Nado Forzado, sumergiéndolos en una piscina cilíndrica durante 6 minutos y registrando el tiempo de inmovilidad. Los ratones sometidos a la prueba de Sujeción de cola fueron distribuidos de la misma manera y administrados con las mismas sustancias para después de 10 días ser suspendidos por el tercio distal de la cola registrándose el tiempo de inmovilidad. Resultados: Los ratones que recibieron el aceite de Plukenetia volubilis a dosis de $1 \mathrm{~g} / \mathrm{kg}$ y $3 \mathrm{~g} / \mathrm{kg}$ presentaron menor tiempo de inmovilidad respecto al control para ambas pruebas, sólo teniendo el grupo con dosis $3 \mathrm{~g} / \mathrm{kg}$ significancia estadística. En el nado forzado el tiempo de inmovilidad con dosis de aceite de $1 \mathrm{~g} / \mathrm{kg}$ y $3 \mathrm{~g} / \mathrm{kg}$ fue 184,7 s y 108,0 s, respectivamente. Para la prueba de Sujeción de cola el tiempo de inmovilidad fue $118,33 \mathrm{~s}$ y $63,33 \mathrm{~s}$ para dosis de $1 \mathrm{~g} / \mathrm{kg}$ y $3 \mathrm{~g} / \mathrm{kg}$ respectivamente. Conclusiones: El aceite de Sacha Inchi administrado por vía oral a dosis de $3 \mathrm{~g} / \mathrm{kg}$ demostró efecto protector similar a fluoxetina, frente a la depresión inducida en los modelos animales empleados.

Palabras clave: depresión, efectos farmacológicos, suspensión de cola, nado forzado

\begin{abstract}
Objective: Evaluate protective effect of Plukenetia volubilis oil (Sacha Inchi) on induced depression in albino mice. Materials and methods: Mice were divided into 4 groups and received the following substances during 10 days: Group $N^{\circ} 1(n=6)$ Vehicle $5 \mathrm{ml} / \mathrm{Kg} / 12 \mathrm{~h}$, Group $\mathrm{N}^{\circ} 2(\mathrm{n}=6)$ : Fluoxetine $10 \mathrm{mg} / \mathrm{Kg} / 24 \mathrm{~h}$, Group $N^{\circ} 3(\mathrm{n}=6)$ : Sacha inchi oil $1 \mathrm{~g} / \mathrm{kg} / 12 \mathrm{~h}$, Group N4 ( $=6$ ): Sacha inchi oil $3 \mathrm{~g} / \mathrm{kg} / 12 \mathrm{~h}$. Then, they were subject to Forced Swimming test, submerging them in a cylindrical pool for 6 minutes and the immobility time was recorded. The mice subjected to the tail suspension test were distributed in the same way and administered with the same substances, after 10 days being suspended by the distal third of the tail, recording the immobility time. Results: The mice that received the Plukenetia volubilis oil at a dose of $1 \mathrm{~g} / \mathrm{kg}$ and $3 \mathrm{~g} / \mathrm{kg}$ had a shorter immobility time regarding control for both tests, and only the group with a dose of $3 \mathrm{~g} / \mathrm{kg}$ had statistical significance. In forced swimming the immobility time with oil doses of $1 \mathrm{~g} / \mathrm{kg}$ and $3 \mathrm{~g} / \mathrm{kg}$ was $184.7 \mathrm{~s}$ and $108.0 \mathrm{~s}$, respectively. For Tail suspension test, the immobility time was $118.33 \mathrm{~s}$ and $63.33 \mathrm{~s}$ for doses of $1 \mathrm{~g} / \mathrm{kg}$ and $3 \mathrm{~g} / \mathrm{kg}$, respectively. Conclusions: Sacha Inchi oil administered orally at a dose of $3 \mathrm{~g} / \mathrm{kg}$ showed a protective effect similar to fluoxetine, against induced depression in the animal model used.
\end{abstract}

Keywords: pharmacologic effects, depression, tail suspention, forced swimming. 


\section{INTRODUCCIÓN}

En el mundo contemporáneo, la carga de trastornos mentales cada vez es mayor, y la depresión es la principal causa de discapacidad a nivel mundial. La Organización Mundial de la Salud (OMS) ha declarado que más de 300 millones de personas viven con depresión, un incremento significativo de más del $18 \%$ entre los años 2005 y 2015. Las personas con trastornos mentales mayormente no cuentan con apoyo, que junto con el miedo al estigma, impiden que muchos puedan acceder al tratamiento requerido para vivir plenamente (1).

El estigma aún percibido en nuestras sociedades para el inicio del tratamiento farmacológico convencional en enfermedades psiquiátricas, conlleva a analizar el efecto protector de diversas sustancias frente a la depresión, dentro de ellas el consumo del aceite de Sacha Inchi que es fuente rica en omega 3. Estos ácidos grasos poliinsaturados tienen acción muy importante en la estructura dinámica y la actividad funcional de las membranas neuronales, en especial en la región sináptica (2). Al aceite de Sacha Inchi no sólo se le atribuyen efectos beneficiosos a nivel del sistema nervioso central, sino también en el sistema cardiovascular, entre otros $(3,4)$.

Los ácidos grasos omega-3 y omega-6 son ácidos grasos poliinsaturados esenciales, no pueden ser sintetizados por el cuerpo humano, por lo deben ser aportados por la dieta (5). La diferencia entre el omega-3 y omega-6 está en la localización del primer doble enlace. El primer representante de los ácidos grasos omega-3 es el ácido a-linolénico (ALA), que se transforma en ácido eicosapentaenoico (EPA) y éste, a su vez, en ácido docosahexaenoico (DHA). En cuanto a los omega-6, su principal representante es el ácido linoleico (LA), siendo uno de sus derivados más importantes, el ácido araquidónico (AA). (2)

El omega-3 es esencial para la formación de las membranas de tejidos como el cerebro, la retina y en las membranas sinápticas están implicados en su estructura dinámica, cambiando su permeabilidad y fluidez, colaborando así en la función sináptica, por lo que un bajo contenido en las membranas neuronales contribuye a un descenso de la transmisión de impulsos nerviosos. $(2,5)$. Además, los efectos positivos de los omega-3 en la depresión dependen de su contenido en el sistema nervioso y de su participación en la neurogénesis y neuroplasticidad, y su propiedad antiinflamatoria contrarrestaría el proceso inflamatorio que ocurre en la depresión. $(2,7)$

Existe evidencia de que pacientes deprimidos presentan una disminución de ácidos grasos omega-3 y, un aumento de los ácidos grasos omega- 6 tanto en las membranas celulares como libres en el plasma. Esta asociación se ha investigado en las membranas plasmáticas de los eritrocitos y se ha encontrado que los pacientes con síntomas depresivos muestran una disminución significativa del omega-3 y una relación
AA/EPA mayor, lo cual está asociado con la severidad de la enfermedad. A mayor relación de AA/EPA se hallaron niveles progresivamente elevados de factor de necrosis tumoral e interleucina 6. $(2,6)$.

Un estudio realizado en China en el año 2012, concluye que el omega 3 DHA muestra propiedades antidepresivas. Además, el DHA tiene múltiples efectos sobre la depresión, incluidos los sistemas de neurotransmisores de monoaminas, las membranas de los glóbulos rojos y en eje hipotálamo-hipófisis-adrenal disminuyendo significativamente los niveles de la hormona adrenocorticotrópica. Todos ellos relacionados con la patogénesis de la depresión (7)

La deficiencia de ácidos grasos omega-3 de cadena larga (EPA y DHA) es un factor que contribuye a la aparición de trastornos depresivos, en parte debido al aumento de la respuesta proinflamatoria. Los omega 3 poseen acción inhibitoria sobre las citoquinas como la interleucina $\beta$ o factor de necrosis tumoral a. Es decir, los omega 3 estarían implicados en la fisiopatología y etiología de la depresión, lo que ofrecería un enfoque terapéutico-preventivo potencial. (5)

Nuestra investigación tiene como objetivo principal comprobar el efecto protector del aceite de Plukenetia volubilis (Sacha Inchi) en la depresión inducida en un modelo animal, evaluando las dosis efectivas del compuesto.

\section{MATERIALES Y MÉTODOS}

En la presente investigación se realizó un estudio tipo analítico. Para la obtención del aceite se usaron las semillas de Sacha Inchi que fueron colocadas en el cilindro de una prensa hidráulica donde se prensó a una presión de 3000 psi. Se decantó los aceites obtenidos y se filtró a través de papel filtro (Whatman N. ${ }^{\circ} 1$ ) con la ayuda de una bomba de vacío (Copelametic, USA), los volúmenes filtrados fueron almacenados. La población estuvo conformada por 48 ratones albinos machos cepas Balb-C (peso $25 \pm 10 \mathrm{~g}$ ) adquiridos del Bioterio del Instituto Nacional de Salud. En la distribución de la muestra, se dividieron aleatoriamente 24 ratones para ser sometidos a prueba de nado forzado; los cuales fueron tratados por vía oral durante 10 días previos de la siguiente manera: Grupo $\mathrm{N}^{\circ} 1(\mathrm{n}=6)$ Agua destilada 5 $\mathrm{ml} / \mathrm{Kg} \mathrm{c} / 12 \mathrm{~h}$, Grupo No $02(\mathrm{n}=6)$ : Fluoxetina $10 \mathrm{mg} / \mathrm{Kg}$ c/24horas, Grupo No $03(n=6)$ : Aceite de sacha inchi 1 $\mathrm{g} / \mathrm{kg} \mathrm{c} / 12$ horas, Grupo No $04(\mathrm{n}=6)$ : Aceite de sacha inchi $3 \mathrm{~g} / \mathrm{kg} \mathrm{c} / 12$ horas. Luego de estos 10 días, fueron sumergidos en una piscina cilíndrica durante 6 minutos y se registró el tiempo de inmovilidad en segundos. La misma distribución y las mismas sustancias fueron administradas durante 10 días para los otros 24 ratones sometidos a la prueba de sujeción de cola, que consistió en suspenderlos por el tercio distal de la cola y registrar el tiempo de inmovilidad en segundos. El procesamiento, producción y análisis del aceite de 
Plukenetia volubilis se realizó en el laboratorio de la Facultad de Farmacia y Bioquímica de la Universidad Nacional Mayor de San Marcos. La administración de las sustancias y la realización de la pruebas de Nado Forzado y Sujeción por la cola se realizó en el laboratorio de la Facultad de Farmacia y Bioquímica de la Universidad San Luis Gonzaga de Ica. Los datos fueron recogidos con ayuda de alumnos de la Facultad de Medicina Daniel Alcides Carrión, quienes desconocían el grupo al que pertenecían los roedores. Posteriormente se procedió a transferir la información a una base de datos elaborada en el programa estadístico SPSS Versión 24.0 Obteniéndose análisis uni, bi y multivariado representados por medio de gráficos y tablas.

\section{RESULTADOS}

COMPOSICIÓN DEL ACEITE DE Plukenetia Volubilis

Tabla 1: Contenido de ácidos grasos del aceite de sacha inchi.

\begin{tabular}{lc}
\multicolumn{1}{c}{ Ácidos grasos } & $\begin{array}{c}\text { Aceite crudo de } \\
\text { Sacha inchi * }\end{array}$ \\
\hline Ácido mirístico (C14:0) & - \\
Ácido palmítico (C16:0) & $3,50 \pm 0,01$ \\
Ácido esteárico (C18:0) & $2,60 \pm 0,01$ \\
Ácido oleico (C18:1) & $10,01 \pm 0,01$ \\
Ácido vacénico & $0,60 \pm 0,01$ \\
Ácido linoléico (C18:2) & $30,00 \pm 0,02$ \\
Ácido a-linolénico (C18:3) & $50,00 \pm 0,02$ \\
Ácido eicosaenoico & $0,20 \pm 0,03$ \\
Ácidos saturados & $7,20 \pm 0,01$ \\
Ácido monoinsaturados & $9,80 \pm 0,02$ \\
Ácido poliinsaturados & $85,24 \pm 0,01$ \\
Relación omega-3/omega & 1,40 \\
\hline
\end{tabular}

Media de dos repeticiones \pm desviación estándar.

En la tabla $\mathrm{N}^{\circ} 1$ describimos los porcentajes de ácidos grasos del aceite de Plukenetia Volubilis (Sacha Inchi) utilizado esta investigación. Cuenta con un $85,24 \%$ de ácidos grasos poliinsaturados, encontrándose en mayor cantidad el ácido linolénico y el ácido linoleico.

PRUEBA DE NADO FORZADO

Tabla 2: Análisis descriptivo del tiempo de inmovilidad en la prueba de nado forzado

\begin{tabular}{lcccccc} 
Grupo $(\mathbf{n}=\mathbf{6})$ & $\begin{array}{c}\text { Tiempo de } \\
\text { inmovilidad }(\mathbf{s})\end{array}$ & $\begin{array}{c}\text { Error } \\
\text { estándar }\end{array}$ & Mínimo & Máximo & P valor* & P valor+ \\
CONTROL & $205,00 \pm 24,29$ & 9,916 & 180 & 240 & - & $\mathrm{p}<0,001$ \\
FLUOXETINA & $130,00 \pm 20,00$ & 8,165 & 100 & 160 & $\mathrm{p}<0,001$ & - \\
P. volubilis D1a & $184,67 \pm 10,33$ & 4,216 & 170 & 200 & $\mathrm{p}=0,244$ & $\mathrm{p}<0,001$ \\
P. volubilis D2b & $108,00 \pm 14,97$ & 6,110 & 90 & 130 & $\mathrm{p}<0,001$ & $\mathrm{p}=0,188$ \\
\hline
\end{tabular}

En la tabla $\mathrm{N}^{\circ} 2$ se muestra los resultados del análisis descriptivo del tiempo de inmovilidad en la Prueba de natación forzada y el $p$ valor comparándolos con el grupo control y con el de fluoxetina. Los ratones tratados con aceite de Plukenetia volubilis (Sacha Inchi) a dosis de 1 $\mathrm{g} / \mathrm{kg}$ y a $3 \mathrm{~g} / \mathrm{kg}$ presentaron tiempos de inmovilidad de 184,67 $\pm 10,33$ y $108,00 \pm 14,97$, respectivamente, los cuales son menores comparados con el grupo control que tuvo un tiempo de inmovilidad 205,00 $\pm 24,29$. Sólo el grupo con dosis de $3 \mathrm{~g} / \mathrm{kg}$ de aceite de Sacha Inchi, tuvo una diferencia con significancia estadística respecto al control. Además, el tiempo de inmovilidad fue menor para los ratones tratados con una dosis $3 \mathrm{~g} / \mathrm{kg}$ de aceite de Sacha Inchi que con el tratamiento antidepresivo estándar (fluoxetina). Los grupos con dosis de $1 \mathrm{~g} / \mathrm{kg}$ y $3 \mathrm{~g} / \mathrm{kg}$ de aceite de Plukenetia volubilis mostraron reducción del tiempo de inmovilidad de $10 \%$ y $47 \%$ respectivamente. 


\section{PRUEBA DE SUJECIÓN DE COLA}

Tabla 3: Análisis descriptivo del tiempo de inmovilidad en la prueba de sujeción de cola

\begin{tabular}{lcccccc} 
GRUPO (n= 6) & $\begin{array}{c}\text { Tiempo de } \\
\text { inmovilidad }\end{array}$ & $\begin{array}{c}\text { Error } \\
\text { estándar }\end{array}$ & Mínimo & Máximo & p valor* & P valor+ \\
CONTROL & $165,83 \pm 17,44$ & 7,12 & 145 & 190 & - & $\mathrm{p}<0,001$ \\
FLUOXETINA & $49,17 \pm 14,29$ & 5,83 & 30 & 70 & $\mathrm{p}<0,001$ & - \\
P. volubilis D1a & $118,33 \pm 22,29$ & 9,09 & 100 & 150 & $\mathrm{p}=0,002$ & $\mathrm{p}<0,001$ \\
P. volubilis D2b & $63,33 \pm 23,38$ & 9,55 & 30 & 90 & $\mathrm{p}<0,001$ & $\mathrm{p}=0,606$ \\
\hline
\end{tabular}

\section{*comparado con el control + comparado el grupo de fluoxetina a Aceite de Plukenetia Volubilis $1 \mathrm{~g} / \mathrm{kg}$ b Aceite de Plukenetia volubilis $3 \mathbf{g} / \mathbf{k g}$}

En la tabla $\mathrm{N}^{\circ} 3$ observamos los resultados del análisis descriptivo del tiempo de inmovilidad en la Prueba de Sujeción de cola y el $p$ valor comparado tanto para el grupo control como el de fluoxetina. Los ratones albinos administrados con aceite de Plukenetia volubilis (Sacha Inchi) a dosis de $1 \mathrm{~g} / \mathrm{kg}$ y a $3 \mathrm{~g} / \mathrm{kg}$ presentaron tiempos de inmovilidad de 118,33 $\pm 22,29$ y 63,33 $\pm 23,38$ segundos, respectivamente, que son menores comparado con el grupo control que tuvo un tiempo de inmovilidad $165,83 \pm 17,44$. Sólo el grupo con dosis de $3 \mathrm{~g} / \mathrm{kg}$ de aceite de Sacha Inchi, tuvo una diferencia con significancia estadística respecto al control. Los grupos con dosis de $1 \mathrm{~g} / \mathrm{kg}$ y $3 \mathrm{~g} / \mathrm{kg}$ de aceite de Plukenetia volubilis mostraron reducción del tiempo de inmovilidad de $28 \%$ y $61 \%$ respectivamente.

\section{DISCUSIÓN}

Desde hace más de medio ciclo, no se han introducido nuevos fármacos, que tengan mecanismos fundamentalmente distintos a los convencionales antidepresivos(8). La indefensión aprendida es un modelo teórico planteado por Seligman, donde estudia el comportamiento animal mientras se encuentre expuesto a estrés incontrolable. Estos animales presentan síntomas comparables con los humanos deprimidos como alteraciones del sueño, disminución de la ingesta y del peso, disminución de la conducta sexual e incremento de la hormona corticotrópica y de la corticosterona. Tanto la prueba de nado forzado como la de Sujeción de cola pertenecen a esta categoría. $(8,9)$ La interpretación de la prueba de Nado forzado la usamos para evaluar si los ratones tienden a adoptar estrategias de afrontamiento activas o pasivas. La administración de fármacos antidepresivos clásicos aumenta la latencia de aparición de inmovilidad y disminuye la duración de ésta, lo cual nos demuestra que el aceite de Plukenetia volubilis (Sacha Inchi) a dosis de 3 $\mathrm{g} / \mathrm{kg}$ se presenta no sólo como factor protector sino que también presentaría potencial utilidad terapéutica.

Una variante de la prueba de natación forzada es la prueba de suspensión por la cola, en la cual se contabiliza el tiempo de inmovilidad, así como la latencia en la aparición de ésta (8). La administración aguda de la mayoría de los antidepresivos reduce la inmovilidad, lo cual fue corroborado en nuestra investigación donde la administración de Fluoxetina, y dosis de $1 \mathrm{~g} / \mathrm{kg}$ y $3 \mathrm{~g} / \mathrm{kg}$ de aceite de Plukenetia volubilis presentaron efecto protector frente a la depresión inducida por estrés agudo.

Investigaciones previas han descrito que la suplementación con DHA durante 50 días en ratones, disminuyó significativamente el tiempo de inmovilidad en la prueba de nado forzado y concluyeron que el DHA tiene múltiples efectos sobre la depresión a través de los neurotransmisores, de las membranas de los eritrocitos y del eje HPA (7).

Otra investigación sobre la efectividad antidepresivos duales en ratones con niveles estrogénicos bajos (10), reportó que este tipo de antidepresivos son efectivos en los machos, pero no para la hembras, motivo por el cuál para no tener variables confusoras dentro de nuestra investigación sólo trabajamos con ratones albinos machos.

Existe múltiples estudios tanto en humanos como en modelos animales que demuestran el beneficio que una dieta balanceada rica en omega 3 o una suplementación con omega 3 traen grandes beneficios durante la gestación y la etapa post parto, ayudando no sólo a la formación del SNC del niño sino previniendo la depresión post parto en la madre $(2,11,12)$. Arbabi et al (12) valoró la asociación de la ingesta de omega 3 y su efecto frente a la depresión atenuando la sintomatogía, concluyendo que la administración de suplementos de omega 3 puede producir un efecto antidepresivo en ratas post parto inducido y que la suplementación también ayudaba a la disminución de los niveles de corticosterona y las citoquinas proinflamatorias.

Se comprobó que el aceite de Plukenetia volubilis posee alto contenido de ácidos grasos poliinsaturados, principalmente ácido linolénico (omega 3). En nuestra investigación se concluyó que el aceite de Plukenetia volubilis (Sacha Inchi) administrado por vía oral a dosis de $3 \mathrm{~g} / \mathrm{kg}$ demostró efecto protector frente a la depresión inducida por los modelos de nado forzado y sujeción de cola. 
En ambas pruebas la fluoxetina obtuvo resultados equivalentes con los obtenidos a dosis de $3 \mathrm{~g} / \mathrm{kg}$ de aceite de Plukenetia volubilis, pero ninguno de los dos mostró superioridad respecto al otro.

Presentamos una alternativa de prevención en la depresión usando el Aceite Sacha Inchi como factor

\section{REFERENCIAS BIBLIOGRÁFICAS}

1. Organización Mundial de la Salud [Internet] n.d. Nota descriptiva: Depresión [Consultado 2018, Enero 10]. Disponible en:

http://www.who.int/mediacentre/factsheets/fs369/es/

2. Gómez Rospide. Ácidos grasos poliinsaturados omega-3 y trastornos depresivos. [Tesis Doctoral] Presentado en Facultad de Farmacia: Universidad Complutense. Julio 2017.

3. Garmendia F, Pando R, Ronceros G. Efecto del aceite de sacha inchi (Plukenetia volúbilis $L$ ) sobre el perfil lipídico en pacientes con hiperlipoproteinemia". Rev Peru Med Exp Salud Publica. 2011;28(4):628-32. Disponible en:

http://www.scielo.org.pe/scielo.php?script=sci_arttext \&pid=S1726-46342011000400009

4. Alayón A, Echeverri I. Sacha inchi (Plukenetia volubilis Linneo): ¿una experiencia ancestral desaprovechada? Evidencias clínicas asociadas a su consumo. Rev. chil. nutr.2016. vol.43 no.2. Disponible en:

https://scielo.conicyt.cl/scielo.php?script=sci_arttext $\&$ pid $=$ S0717-75182016000200009

5. Soborg Husted K, Bouzinova E. The importance of $n-6 / n-3$ fatty acids ratio in the major depressive disorder. Medicina. 2016. (52) 139-147. Disponible en: https://www.sciencedirect.com/science/article/pii/S10 10660X16300210?via\%3Dihub

6. Tapia A, Masson L. Niveles de ácidos grasos poliinsaturados omega-3 en membranas de protector basándonos en modelos animales sometidos a estrés inducido, abriendo así paso hacia futuras investigaciones farmacológicas en nuestro país y región de tratamientos distintos y prevención de la depresión con productos naturales.

eritrocitos de mujeres con depresión. Rev Chil Nutr.2008. Vol. 35, N4, 406-412. Disponible en: https://scielo.conicyt.cl/scielo.php?script=sci_arttext \&pid=S0717-75182008000500002

7. Jiang L. Liang Q, Shi Y. Pure docosahexaenoic acid can improve depression behaviors and affect HPA axis in mice. EurRevMedPharmacolSci. 2012; 16: 1765-1773

8. Balada F, Márquez C, Nadal R, Redolar D, Silvestre J. Farmacología y Endocrinología del comportamiento.2012. [Primera edición].Editorial UOC. 212-217

9. Cassano P, Argibay P. La enfermedad depresiva y sus modelos animals. Rev. Hosp. Ital. B.Aires.2009.

10. Castellanos Cruz L. Comparación del Efecto Tipo Antidepresivo de Fluoxetina y Duloxetina entre Ratas Macho y Hembra [Tesis de Maestría]. Centro de Investigación y de Estudios avanzados del instituto politécnico nacional sede sur; 2013.

11. Tapia A. Ácidos grasos omega-3 para la prevención y tratamiento de las depresiones en el embarazo y post parto. Rev Chil Obstet Ginecol. 2004, 69(5): 399-403

12. Arbabi L, Baharuldin M, Moklas M, Fakurazi $S$, Muhammad S. Antidepressant-like effects of omega-3 fatty acids in postpartum model of depression in rats. Behavioural Brain Research. 2014;271:65-71.

\section{Las ediciones anteriores de revista médica PANACEA están disponibles en:}

\section{www.revpanacea.unica.edu.pe}

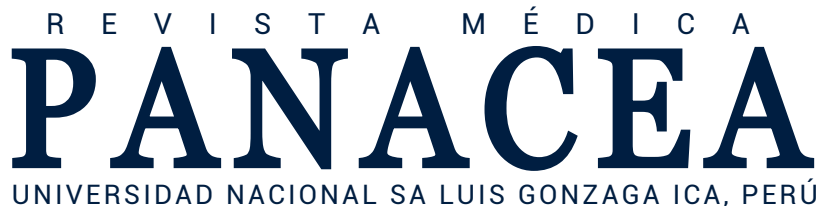

